\title{
A Multicriteria Decision Making Environment for Engineering Design and Production Decision-Making
}

\author{
A. Mosavi \\ University of Debrecen, Hungary
}

\begin{abstract}
A novel environment for optimization, analytics and decision support in general engineering design problems is introduced. The utilized methodology is based on reactive search optimization (RSO) procedure and its recently implemented visualization software packages. The new set of powerful integrated data mining, modeling, visualiztion and learning tools via a handy procedure stretches beyond a decisionmaking task and attempts to discover new optimal designs relating to decision variables and objectives, so that a deeper understanding of the underlying problem can be obtained. In an optimal engineering design environment as such solving the multicriteria decision-making (MCDM) problem is considered as a combined task of optimization and decisionmaking. Yet in solving real-life MCDM problems often most of attention has been on finding the complete Pareto-optimal set of the associated multiobjective optimization (MOO) problem and less on decision-making. In this paper, along with presenting two case studies, the proposed interactive procedure which involves the decision-maker (DM) in the process addresses this issue effectively. Moreover the methodology delivers the capablity of handling the big data often associated with production decision-making as well as materials selection tasks in engineering design problems.
\end{abstract}

\section{Keywords:}

Opimal engineering design, interactive multicriteria decision making, reactive search optimization, multiobjective optimization

\section{INTRODUCTION}

The MCDM environments [1,3,4,5,6,18,19,32,81,84,85] build their bases on software tools used for a large number of applications from modeling, optimization and decisionmaking tasks, to performance's simulation. Further addition of new tools is intended to extend the support to the creative part of the design process and also the capability to deal with big data $[2,10,34,35,59]$. This support empower the designers to improve the performance of their concepts, allowing computers to take part on the generation of variants, and on the judgment, by true modeling of these variants. Integration of data mining, modeling, learning, and interactive decisionmaking are all parts of a reliable software tool that can nurture the knowledge of designers to generate new solutions, based on many separate ideas leading to new the design concepts $[7,8,9,12,23]$.

The methods for structural and topological optimal design, based on evolutionary design, currently are widely used to obtain optimal geometric solutions [15,16,21,22,24,37,59]. The methods and design stragey as such are evolving to configurations that minimize the cost of trial and error and perform far beyond the abilities of the most skilled designers. Although in developing a multicriteria decision making environment relying only on evolutionary design components, in today's ever-increasing complexity when often numerous design objevtives involved, is not sufficient $[14,23,17,60]$.
Moreover most studies in the past concentrated in finding the optimum corresponding to a single goal, say designing for minimum cost or maximum quality. The single-obective optimization procedure searches through possible feasible solutions and at the end identifies the best solution. Often, such solution lacks the consideration of other important design objectives. Fortunately applied optimization over the years have been dramaticaly changed, particularly with the availability of efficient MCDM algorithms which facilitates a DM to consider more than one conflicting goals simultaneously [18].

The task of MCDM is divided into two parts: (1) a MOO procedure to discover conflicting design trad-offs and (2) a decision-making process to choose a single preferred solution among them [18]. Although both processes of optimization and decision-making are considered as two joint tasks, yet they are often treated as a couple of independent activities $[12,18]$. For instance evolutionary multiobjective optimization (EMO) algorithms $[15,16]$ have mostly concentrated on the optimization aspects i.e. developing efficient methodologies of finding a set of Pareto-optimal solutions. However finding a set of trade-off optimal solutions is just half the process of optimal design in a multicriteria decision making environment. This has been the reason why EMO researchers were looking to find ways to efficiently integrate both optimization and decision making tasks in a convenient way $[9,14,17,23,60]$ where the efficient MOO algorithms facilitate the DMs to consider multiple and conflicting goals of a MCDM problem simultaneously. Some examples of such algorithms and potential applications could be found in $[25,26,27,28,29,30,37,59]$. Nevertheless within the known approaches to solving complicated MCDM problems there are different ideologies and considerations in which any decisionmaking task would find a fine balance among them.

In traditional applications to MCDM $[12,18]$ often the single optimal solution is chosen by collecting the DM's preferences where MOO and decision-making tasks are combined for obtaining a point by point search approach [11,12]. In addition in MOO and decision-making, the final obtained solutions must be as close to the true optimal solution as possible and the solution must satisfy the preference information. Towards such a task, an interactive DM tool to consider decision preferences is essential. This fact has motivated novel researches to properly figure out the important task of integration between $\mathrm{MOO}$ and MCDM $[14,17,22,23,60]$. Naturally in MCDM, interactions with the DM can come either during the optimization process, e.g. in the interactive EMO optimization $[15,23,60]$, or during the decision-making process [16,37]. In fact there exists a number of interactive MOO methods in the MCDM literature [7,11].

\section{A REVIEW}

The usage of EMO in real-life optimal design has been always an important interest to MCDM community. In the EMObased optimal design there are two different ways identified by which EMO and MCDM methodologies can be combined 
together [23]. Either EMO followed by MCDM or, MCDM integrated in an EMO. In the first way, an EMO algorithm is applied to find the Pareto-front solutions. Afterward, a single preferred solution is chosen from the obtained set by using a MCDM procedure. In this way EMO application helps a DM to analyze different trade-off solutions to choose the final one. However the DM has to go through analyzing many different solutions to be able to make the final decision. Therefore the DM has to consider too many possible solutions. As the typical DM cannot deal with more than a very limited number of information items at a time [63] the methods as such are reported inefficient [23].

Alternatively a MCDM procedure could be integrated within an EMO to find the preferred Pareto-front solutions where the search is concentrated on the important region of the Paretofront [14]. This would let the optimization task to evaluate the preferences of the DM interactively. These approaches to interactive evolutionary algorithms are reviewed in [17]. Additionaly a summary can be found in the text by Miettinen [18]. Further popular approaches as such include interactive surrogate worth trade-off method [19], the reference point method [20] and the NIMBUS approach [12].

All above procedures require a DM to provide the design preferences [14]. A search workflow is then used to find the optimum of the objective task. This procedure is repeated many times until the DM is satisfied with the obtained final solution. For instance in $[21,22]$ an EMO procedure is applied to a complicated design problem and then an interactive methodology is employed to choose a single solution. In [23], EMO is combined with MCDM procedures, and an interactive procedure is suggested where the EMO methodologies are combined with a certain and efficient MCDM technique. The work later in [23] was extended by involving more MCDM tools and integrations with further software packages such as MATLAB, for providing better working on more real-life study case [14]. In [23] unlike the classical interactive methods in [12], a good estimation of the Pareto-optimal frontier is created, in which helps to concentrate on a particular region. The authors in [23] conclude that when an approach is best suited for one problem it may be inadequate in another problem. As the result worth mentioning that in developing MCDM and EMO novel integrations, a successful procedure could include more than one optimization and decision-making tool in it so that any number of optimization and decision-making tool may be combined to build an effective problem solving procedure [24]. The researches reviewed above, have motivated other EMO, MCDM and optimal design researches, including our article, to develop such integration schemes further by considering other potential optimization and decision-making tools.

\subsection{Drowbacks to Solving MOO Problems with EMO Algorithms}

The general form of a MOO problem [7,8], can be stated as; Minimize $\mathbf{f}(\boldsymbol{x})=\left\{f_{\mathbf{1}}(\boldsymbol{x}), \ldots, f_{\boldsymbol{m}}(\boldsymbol{x})\right\}$, Subjected to $\boldsymbol{x} \in \Omega$, where $\mathbf{x} \in \mathbb{R}^{n}$ is a vector of $n$ decision variables; $\mathbf{x} \subset \mathbb{R}^{\mathrm{n}}$ is the feasible region and is specified as a set of constraints on the decision variables; $\mathbf{f}: \Omega \rightarrow \mathbb{R}^{m}$ is made of $m$ objective functions subjected to be minimization. Objective vectors are images of decision vectors written as $\mathbf{z}=\mathbf{f}(\boldsymbol{x})=$ $\left\{f_{1}(\boldsymbol{x}), \ldots, f_{m}(\boldsymbol{x})\right\}$. Yet an objective vector is considered optimal if none of its components can be improved without worsening at least one of the others. An objective vector $\mathbf{z}$ is said to dominate $\mathbf{z}^{\prime}$, denoted asz $\prec \mathbf{z}^{\prime}$, if $z_{k} \leq z_{k}^{\prime}$ for all $k$ and there exist at least one $h$ that $z_{h} \leq z_{h}^{\prime}$. A point $\widehat{\boldsymbol{x}}$ is Pareto optimal if there is no other $\boldsymbol{x} \in \Omega$ such that $\mathbf{f}(\boldsymbol{x})$ dominates $\mathbf{f}(\widehat{\boldsymbol{x}})$. The set of Pareto optimal points is called Pareto set (PS). And the corresponding set of Pareto optimal objective vectors is called Pareto front (PF).

The EMO algorithms $[16,24,25]$ for solving MCDM problems have been around for up to two decades now, and are well suited to search for a set of PS to be forwarded to the DM. Considering solving MCDM problems, EMO algorithms are among the most popular a posteriori methods for generating PS of a MOO problem aiming at building a set of points near the PF. However they become inefficient for increasing number of objectives. MOO of curve and surfaces [37,59] would be a good example for such an ineffective attempt due to increasing complexity. Because the proportion of PF in a set grows very rapidly with the dimension $\boldsymbol{m}$, therefore the former approaches for solving the MOO of the curve and surfaces [13, 37,59] whether a priori or a posteriori, in particular EMO, would involve plenty of various complications. In fact the reality of applied optimal design has to consider plenty of priorities and drawbacks to both interactive and non-interactive approaches. Although the mathematical representative set of the MCDM model is often created however presenting a human DM with numerous representative solutions on a multi-dimensional PF is way complicated. This is because the typical DM cannot deal with more than a very limited number of information items at a time [63]. Therefore an improved decision procedures should be developed according to human memory and his data processing capabilities. In addition often DMs cannot formulate their objectives and preferences at the beginning. Instead they would rather learn on the job. This is already recognized in the optimal design formulation, where a combination of the individual objectives into a single preference function is not executed. Considering the problems in $[27,37,59]$ the $\mathrm{DM}$ is not clear about the preference function. This uncertainty is even increased when the objectives such as beauty involved. This fact would employ lots of uncertainty and inconsistency.

Consequently interactive approaches $[12,14,18,23]$ try to overcome some of these difficulties by keeping the user in the loop of the optimization process and progressively focusing on the most relevant areas of the PF directed by DM. This is done when the fitness function is replaced by a human user. However most DMs are typically more confident in judging and comparing than in explaining. They would rather answer simple questions and qualitative judgments to quantitative evaluations. In fact the identified number of questions that has to be asked from the DM a crucial performance indicator of interactive methods. This would demand for selecting appropriate questions, for building approximated models which could reduce bothering the DM $[33,60]$.

The above facts, as also mentioned in [39], and later in [36] demand a shift from building a set of PF, to the interactive construction of a sequence of solutions, so called braincomputer optimization [60], where the DM is the learning component in the optimization loop, a component characterized by limited rationality and advanced questionanswering capabilities. This has been the reason for the systematic use of machine learning techniques for online learning schemes in optimization processes available in the software architectures of LIONsolver and Grapheur $[39,40,60]$. 


\section{BRAIN-COMPUTER OPTIMIZATION (BCO) APPROACH TO STOCHASTIC LOCAL SEARCH}

As Battiti et al. [8,39], also clearly state, the aim of stochastic local search is to find the minimum of the combinatorial optimization function $f$, on a set of discrete possible input values $X$. To effectively and interactively doing so the focus in [60] is devoted to a local search, hinting at reactive search optimization (RSO) with internal self-tuning mechanisms, and $\mathrm{BCO}$ i.e. a $\mathrm{DM}$ in the interactive problem-solving loop. Accordingly in this context the basic problem-solving strategy would start from an initial tentative solution modifying the optimization function. According to [8] the local search starts from a configuration of $X(0)$ and builds a search trajectory $X^{(0)}, \ldots, X^{(t+1)}$. where $X$ is the search space and $X^{(t)}$ is the current solution at iteration $t$, time. Then $N\left(X^{(t)}\right)$ would be the neighborhood of point $X^{(t)}$,obtained by applying a set of basic moves $\mu_{0}, \mu_{1}, \ldots, \mu_{M}$ to the configuration of $N\left(X^{(t)}\right)=\left\{X \in X\right.$ Such that $X=\mu_{i}\left(X^{(t)}\right), i=0, \ldots$, $M\}$. If the search space is given by binary strings with a given length $L: X=\{0,1\}^{L}$, the moves can be those changing the individual bits, and therefore $L$ is equal to the string length $M$. The accuracy of the achieved point is a point in the neighborhood with a lower value of $f$ to be minimized. The search then would stop if the configuration reaches a local minimum [7].

$$
\begin{aligned}
& \mathrm{Y} \leftarrow \quad \text { IMPROVING-NEIGHBOR }\left(N\left(X^{(t)}\right)\right) \\
& X^{(t+1)}=\left\{\begin{array}{cc}
Y & \text { if } f(Y)<X^{(t)} \\
X^{(t)} & \text { otherwise (search stops) }
\end{array}\right.
\end{aligned}
$$

Here the local search works very effectively and the improving-neighbor returns an improving element in the neighborhood. This is manily because most combinatorial optimization problems have a very rich internal structure relating the configuration $X$ and the $f$ value [8]. In the neighborhood the vector containing the partial derivatives is the gradient, and the change of $f$ after a small displacement is approximated by the scalar product between the gradient and the displacement [38].

\subsection{Learning Component; DM in the Loop}

In problem-solving methods of stochastic local search, proposed in [60], where the free parameters are tuned through a feedback loop, the user is considered as a crucial learning component in which different options are developed and tested until acceptable results are obtained. As explained in [7] by inserting the machine learning the human intervention is decreased by transferring intelligent expertise into the algorithm itself. Yet in order to optimize the outcome setting the parameters and observing the outcome, a simple loop is performed where the parameters in an intelligent manner changed until a suitable solution is identified. Additionaly to operate efficiently, RSO uses memory and intelligence, to recognize ways to improve solutions in a directed and focused manner.

In the RSO approach of problem solving the brain-computer interaction is simplified. This is done via learning-optimizing process which is basically the insertion of the machine learning component into the solution algorithm. In fact the strengths of RSO are associated to the brain charactristics i.e. learning from the past experience, learning on the job, rapid analysis of alternatives, ability to cope with incomplete information, quick adaptation to new situations and events
[7,8]. Moreover the term of intelligent optimization in RSO refers to the online and offline schemes based on the use of memory, adaptation, incremental development of models, experimental algorithmics applied to optimization, intelligent tuning and design of heuristics. In this context with the aid of advanced visualization tools implemented within the software architecture packages $[39,40,60]$ the integration of visualization and automated problem solving and optimization would be the centere of attention.

\subsection{RSO and Visualization Tools; an Effective Approach to MCDM}

Visualization is an effective approach in the operations research and mathematical programming applications to explore optimal solutions, and to summarize the results into an insight, instead of numbers [31, 32]. Fortunately during past few years, it has been a huge development in combinatorial optimization, machine learning, intelligent optimization, and RSO [7,8], which have moved the advanced visualization methods even further. Previous work in the area of visualization for MCDM [32] allows the DM to better formulate the multiple objective functions for large optimization runs. Alternatively in our research utilizing RSO and visualization [60], which advocates learning for optimizing, the algorithm selection, adaptation and integration, are done in an automated way and the user is kept in the loop for subsequent refinements. Here one of the crucial issue in MCDM is to critically analyzing a mass of tentative solutions assusiated with big data, which is visually mined to extract useful information [34,35,36]. In developing RSO in terms of learning capabilities there has been a progressive shift from the DM to the algorithm itself, through machine learning techniques [7].

Concerning solving the MCDM problems, utilizing RSO, the final user is not distracted by technical details, instead concentrates on using his expertise and informed choice among the large number of possibilities. Algorithms with selftuning capabilities like RSO make life simpler for the final user. To doing so the novel approach of RSO is to integrate the machine learning techniques, artificial intelligence, reinforcement learning and active learning into search heuristics. According to the original literature [33] during a solving process the alternative solutions are tested through an online feedback loop for the optimal parameters' tuning. Therefor the DM would deal with the diversity of the problems, stochasticity, and dynamicity more efficiently. Here are some case studies treated very promising by RSO $[26,27,28,29,36]$. Worth mentioning that RSO approach of learning on the job is contrasted with off-line accurate parameter tuning $[39,40]$ which automatically tunes the parameter values of a stochastic local search algorithm.

\subsection{Characteristics of the Proposed Approach}

During the process of solving the real-life problems exploring the search space, utilizing RSO, many alternative solutions are tested and as the result adequate patterns and regularities appear $[39,40]$. While exploring, the human brain quickly learns and drives future decisions based on the previous observations and searching alternatives. For the reason of rapidly exploiting the most promising solutions the online machine learning techniques are inserted into the optimization engine of RSO [8]. Furthermore with the aid of inserted machine learning a set of diverse, accurate and crucial alternatives are offered to the DM. The complete series of solutions are generated. After the exploration of the design 
space, making the crucial decisions, within the multiple existing criteria, totaly depends on several factors and priorities which are not always easy to describe before starting the solution process. In this context the feedbacks from the DM in the preliminary exploration phase can be considered so that a better arrangement of the parameters takes the preferences into account [7]. Further relevant characteristics of RSO, according to [8], could be summarized as; learning on the job, rapid generation, and analysis of many alternatives, flexible decision support, diversity of solutions and anytime solutions.

\subsection{Applications}

A number of complex optimization problems arising in widely different contexts and applications which has been effectively treated by the general framework of RSO are reviewed in [36]. This include the real-life applications in computer science and operations research community combinatorial tasks, applications in the area of neural networks related to machine learning and continuous optimization tasks. This would include risk management, managing the big data of social networks, transportation, healthcare, marketing and ecommerce. In addition in the following we summarize some applications in real-life engineering application areas which are the main interests of this research. In the area of electric power distribution there have been reported a series of reallife applications [42]. An open vehicle routing problem [43], as well as the pickup and delivery problem [44] both with the time and zoning constraints is modeled where the RSO methodology is applied to the distribution problem in a major metropolitan area. Alternatively to solve the vehicle routing problem with backhauls a heuristic approach based on a hybrid operation of reactive tabu search is proposed in [45]. By utilizing the RSO the flexible job-shop scheduling [46], the plant location problem [47], the continuous flow-shop scheduling problem [48], adaptive self-tuning neurocontrol [49] and the real-time dispatch of trams [50] were effectively solved. Moreover various applications of RSO focused on problems arising in telecommunication networks, internet and wireless in terms of optimal design, management and reliability improvements e.g. [51]. The multiple-choice multidimensional knapsack problem with applications to service level agreements and multimedia distribution is studied in [52]. In the military related applications, in optimal designing of an unmanned aerial vehicle routing system [53] and in finding the underwater vehicle trajectories [54], RSO worked wonder. The problem of active structural acoustic control [55] and visual representation of data through clustering [56] are also well treated. Additionally the solution of the engineering roof truss design problem is discussed in [57]. An application of RSO for designing barrelled cylinders and domes of generalized elliptical profile is studied in [58]. Overall a series of successful projects accomplished with the aid of RSO could be found in $[25,26,27,28,29,30,36]$. Further applications of RSO are listed in $[33,40]$ and the stochastic local search book [41].

\subsection{Software Architecture Packages for the Proposed Reactive and Interactive MCDM Environment}

Grapheur and LIONsolver [7,8,39,40] are two implementaions of RSO. The software implements a strong interface between a generic optimization algorithm and DM. While optimizing the systems produce different solutions, the $\mathrm{DM}$ is pursuing conflicting goals, and tradeoff policies represented on the multi-dimensional graphs $[33,40]$. During multi-dimensional graphs visualization in these software packages, it is possible to call user-specific routines associated with visualized items. This is intended as the starting point for interactive optimization or problem solving attempts, where the user specifies a routine to be called to get information about a specific solution. These implementations of RSO are based on a three-tier model, independent from the optimization algorithm, effective and flexible software architecture for integrating problem-solving and optimization schemes into the integrated engineering design processes and optimal design, modeling, and decision-making.

For solving problems with a high level of complexity, modeling the true nature of the problem is of importance and essential. For this reason a considerable amount of efforts is made in modeling the MOO problems in Scilab which later will be integrated into optimizer package. Here, as an alternative to the previous approaches $[22,23,24]$ the robust and interactive MOO algorithm of RSO is proposed in order to efficiently optimize all the design objectives at once in which couldn't be completely considered in the previous attempts. In this framework the quality of the design, similar to the previous research workflows, is measured using a set of certain functions. Then an optimization algorithm is applied in order to optimize the function to improve the quality of the solution. Once the problem is modeled in scilab it is integrated to the optimizer via advanced interfaces to the RSO algorithm and its brain-computer implementations and visualizations. In this framework the application of learning and intelligent optimization and reactive business intelligence approaches in improving the process of such complex optimization problems is accomplished. Furthermore the problem could be further treated by reducing the dimensionality and the dataset size, multi-dimensional scaling, clustering and visualization tools $[36,60]$.

\section{CASE STUDY 1: WELDED BEAM DESIGN}

The problem of welded beam design is a well-known case study in structural engineering, dealing with optimal designing the form of steel beams and with connecting them to form complex stuctures [24]. This case study has been used by many experts as a benchmark problem of single and also multiobjective design optimization. The problem of optimal designing a welded beam consists of dimensioning a welded steel beam and the welding length in order to minimize the cost subjected to bending stress, constraints on shear stress, the buckling load on the bar, the end the deflection of the beam, and side constraints. There are four design variables i.e. $h, l, t, b$ shown in the Fig. 1. Structural analysis of the welded beam leads to two nonlinear objective functions subjected to five nonlinear and two linear inequality constraints. The objectives include the fabrication cost and the end deflection of the beam. In our case, the aim is to reduce fabrication cost without causing a higher deflection. Decision-making on the preferred solution among the Pareto-optimal set requires the intelligent participation of the designer, to identify the tradeoff between cost and deflection.

As it is shown in the figure 1 the beam is welded on another beam carrying a certain load $\mathrm{P}$. The problem is well studied as a single objective optimization problem [24], but we have transformed the original single objective problem into a twoobjective problem for more flexible design. In the original study the fabrication cost $\left(f_{1}(\mathrm{x})\right)$ of the joint is minimized with four nonlinear constraints related to normal stress, shear stress, buckling limitations and a geometry constraint. 


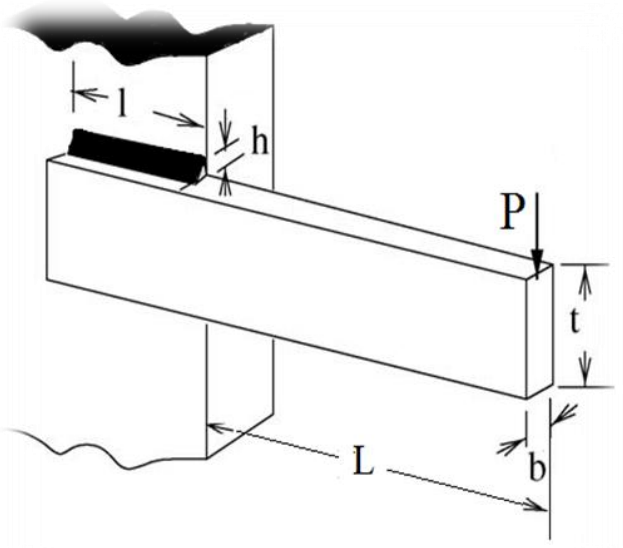

Fig 1: The welded beam optimal design problem.

With the following formulation we have introduced one more objective i.e. minimization of the end deflection $(\delta(x))$ of the structure. The problem has four decision variables presented in the optimization formulation, i.e. thickness of the beam $b$, width of the beam $t$, length of weld $l$, and weld thickness $h$. The overhang portion of the beam has a length of 14 in and $F$ $1 / 4$ 6; $000 \mathrm{lb}$ force is applied at the end of the beam. The mathematical formulation of the problem is given as;

Minimize $f_{1}(\mathrm{x})=1.104711 h^{2} l+0.04811 t b(14.0+l)$,

Minimize $f_{2}(\mathrm{x})=\delta(x)=\frac{2.1952}{t^{3} b}$

Subjected to $g_{1}(\mathrm{x}) \equiv 13,600-\tau(\mathrm{x}) \geq 0$,

$$
\begin{aligned}
& g_{2}(\mathrm{x}) \equiv 30,000-\sigma(\mathrm{x}) \geq 0, \\
& g_{3}(\mathrm{x}) \equiv b-h \geq 0 \\
& g_{4}(\mathrm{x}) \equiv P_{c}(\mathrm{x})-6,000 \geq 0
\end{aligned}
$$

$0.125 \leq h, \quad b \leq 5.0, \quad 0 . \leq l, \quad t \leq 10.0$

The described problem has recently been modeled and solved utilizing a novel optimal design strategy so called interactive multi-objective optimization and decision-making using evolutionary methods (I-MODE). However I-MODE software implementation can only consider a maximum of three objectives due to limitation of visual representation of the Pareto-optimal solutions. In applying I-MODE framework to further real-life optimal design problems $[22,23,25,26,27,28,29,30]$ in which real DMs are involved there exists a number of shortcomings which are considered and improved in the proposed RSO optimal design strategy.

\subsection{Creating the Model in Scilab}

Scilab is now a robust, flexible and low-cost alternative to MATLAB which makes is an ideal modeling tool to be integrated to the MCDM. The success story presented in this paper in a short time and on a limited budget is the evidence of this statement. In fact he ongoing global crisis started in 2008 has forced the design companies to focus on efficiency and costs reduction by exploring open source software tools as a possible alternative to closed source. Moreover the final integrated optimal design tool has a fast and efficient computational capabilities in addition to the possibilities to automatically call parallel instances of the Scilab routine in background batch mode.

Here in this case study Scilab file contains a string definition, i.e. g name, inluding a short, mnemonic name for the model as well as two 8-bit integers, i.e. g_dimension and g_range, defining the number of input and output variables of the model. Additionaly the file has two real-valued arrays; i.e. g_min and g_max, containing the minimum and maximum values allowed for each of the input and output variables. The following description is a simple definition of a function that is integrated to RSO so it can be understood and utilized by software implementation $[7,8,40]$.

The extensive implementation of the problem in Scilab is

\begin{tabular}{|c|c|c|}
\hline \multicolumn{3}{|c|}{$\begin{array}{l}\text { Choose direction of optimization } \\
\text { Choose desirable target outputs }\end{array}$} \\
\hline \multirow{2}{*}{$\begin{array}{l}\text { fabrication cost ( } f 1 \text { ) } \\
\text { end deflection ( } f 2 \text { ) }\end{array}$} & Minimize & $\checkmark$ \\
\hline & Minimize & $\rightarrow$ \\
\hline \multirow{2}{*}{$\begin{array}{l}\mathrm{f} 1 \text { with penalty } \\
\mathrm{f} 2 \text { with penalty }\end{array}$} & Minimize & $\checkmark$ \\
\hline & Minimize & $\checkmark$ \\
\hline Penalty & Minimize & $\checkmark$ \\
\hline \multicolumn{3}{|c|}{ [ - ] Constraints on inputs } \\
\hline \multicolumn{3}{|l|}{ welding depth (h) } \\
\hline Min & \multicolumn{2}{|c|}{0.125} \\
\hline Max & \multicolumn{2}{|c|}{5.0} \\
\hline \multicolumn{3}{|l|}{ welding length (I) } \\
\hline Min & \multicolumn{2}{|c|}{0.1} \\
\hline Max & \multicolumn{2}{|c|}{10.0} \\
\hline \multicolumn{3}{|l|}{ height (t) } \\
\hline Min & \multicolumn{2}{|c|}{0.1} \\
\hline Max & \multicolumn{2}{|c|}{10.0} \\
\hline \multicolumn{3}{|l|}{ thickness (b) } \\
\hline Min & \multicolumn{2}{|c|}{0.125} \\
\hline Max & \multicolumn{2}{|c|}{5.0} \\
\hline
\end{tabular}
available in [36].

$$
\begin{aligned}
& \text { g_name = "ZDT1"; } \\
& \text { g_dimension = int } 8(2) ; \\
& \text { g_range = int } 8(2) ; \\
& g \_ \text {min }=[0,0,0,0] ; \\
& g \_ \text {max }=[1,1,1,1] ; \\
& \text { g_names = ["xl", "x2", "f1", "f2"]; } \\
& \text { function } f=g \_f u n c t i o n(x) \\
& f 1 \_x 1=x(1) \\
& g \_x 2=1+9 * x(2) \\
& h=1-\operatorname{sqrt}\left(f 1 \_x 1 / g \_x 2\right) \\
& f=\left[1-f 1 \_x 1,1-g \_x 2 * h\right] \\
& \text { endfunction; }
\end{aligned}
$$

Fig 2: Description of the welded beam design problem in the software architecture of RSO multiobjective optimization; tuning the objectives and constraints

Among the four constraints, $g_{1}$ deals with the shear stress developed at the support location of the beam which is meant to be smaller than the allowable shear strength of the material $(13,600 \mathrm{psi})$. The $g_{2}$ guarantees that normal stress developed 
at the support location of the beam is smaller than the allowable yield strength of the material (30,000 psi). The $g_{3}$ makes certain that thickness of the beam is not smaller than the weld thickness from the standpoint. The $g_{4}$ keeps the allowable buckling load of the beam more than the applied load P for safe design. A violation of any of the above four constraints will make the design unacceptable. More on adjusting the constraints would be availabe in [22,23]. Additionaly on the stress and buckling terms calculated in [24] needless mentionning that they are highly non-linear to design variables.

\subsection{Setting up the RSO Software}

Here the RSO software architecture of LIONsolver [33,40] helps the designer to become aware of the different posibilities and focus on his preferred solutions, within the boundary of constraints. Consequently the constraints are transformed into a penalty function which sums the absolute values of the violations of the constraints plus a large constant. Unless the two functions are scaled, the effect of deflection in the weighted sum will tend to be negligible, and most Pareto-optimal points will be in the area corresponding to the lowest cost. Therefore each function is devided by the estimated maximum value of each function in the input range [24]. The Pareto-optimal solutions of the multiobjective optimization and MCDM corresponding to fabrication cost vs. end deflection of the beam are visualy presented in the graph of Figure 3.

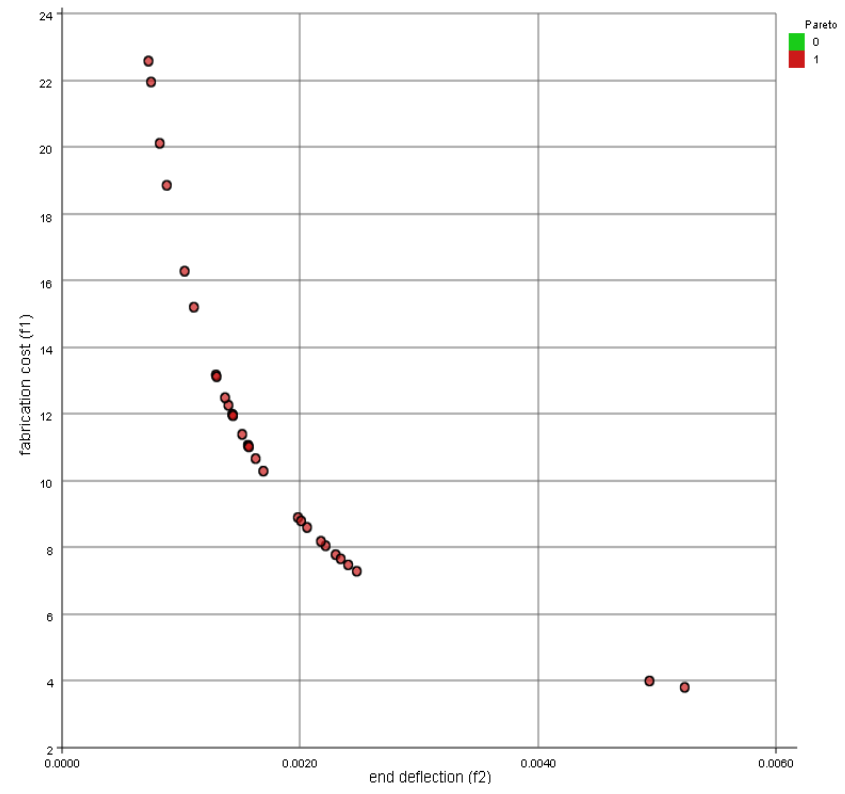

Fig 3: Set of pareto-optimal solutions, fabrication cost vs. end deflection of the beam

By associating a multidimentional graph for an advanced visualization, available in Figure 4, and a paralell chart, available in Figure 5, to the results table, the MCDM problem very clearly comes to the consideartion and the final decision is very confidently made. Here as the result, quite similar to the results abtained from the other approach in [23] it is observed that the welding length $l$ and depth $h$ are inversely proportional, the shorter the welding length, the larger the depth has to be, and that height $t$ tends to be close to its maximum allowed value.

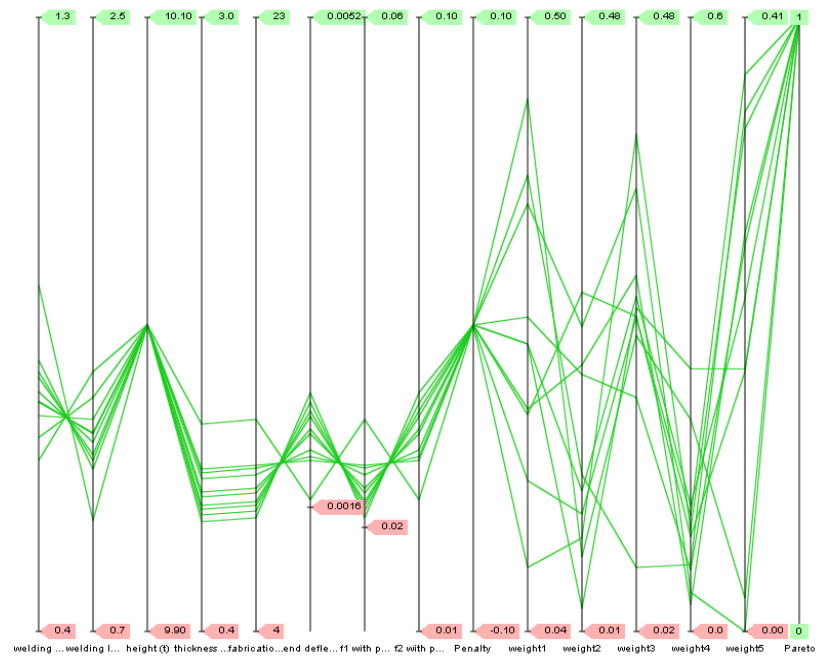

Fig 4: Parelel chart including all variables, constraints and optimization objectives

The final visualization and observations can inspire many problem simplifications e.g. it is observed that by fixing the height to its maximum value and by expressing the length as a function of depth, therefore eliminating two variables from consideration in the future explorations, the optimal design problem would be simpler.

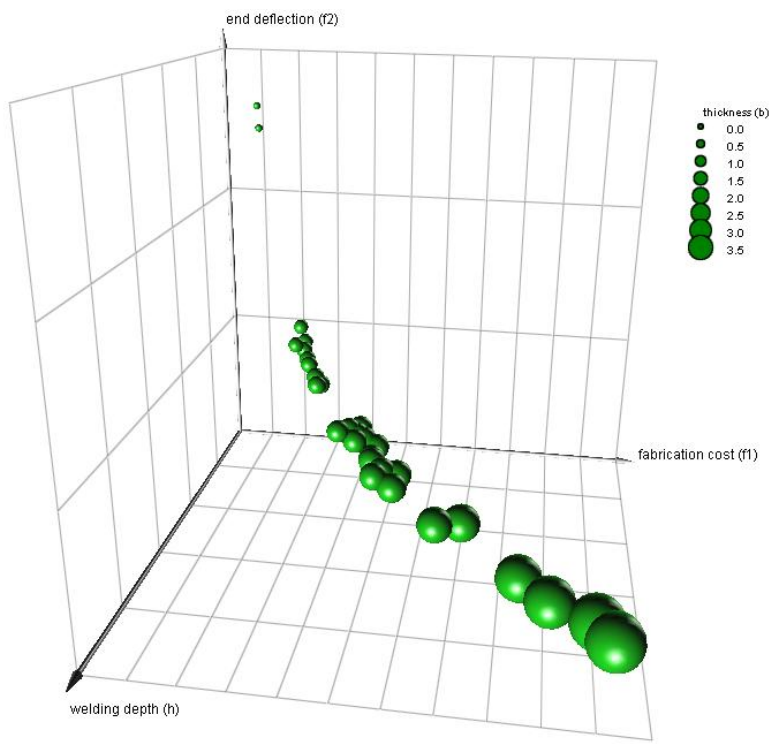

Fig 5: Multidimentional graph for an advanced visualization; the fabrication cost vs. end deflection of the beam

\section{CASE STUDY 2. OPTIMAL DESIGN OF TEXTILE COMPOSITES AND MATERIAL SELECTION}

The second case study would be dealing with optimal design of textile composites, a more challenging task where the number of design criteria are increased and the geometry becomes way complicated. Textile composite materials consist of a polymer matrix combined with textile reinforcement. Typical applications range from high performance aerospace components to structural parts of transportation industry. In fact because of the numerous 
advantages of composites in comparison to traditional materials there has been an increasing trend in the usage of composite materials in different industries.

Former research on optimal design of textile composites [75] highlighted that the ability to test preliminary designs is not economically workable and the assesment of preliminary materials systems urges the use of simulation tools. Such a strategy would improve the process of multi-criteria materials selection [76] and also can empower designers in considering the role of materials selection in design of materials and products. Jahan and Edwards believe [78] that there appears to be a simulation-based materials design revolution underway in which materials selection could be improved in order to more rapidly qualify new material designs. This would happen by shift from costly and time-consuming physical experimentation to less costly computational modeling and design [77].

The integrated and multi-disciplinary design process of composites has been very challenging. The design process is divided into several criteria and sub-criteria, while receiving the contributions of many different departments trying to meet conflicting requirements of the design simultaneously. Consequently, an optimal design process within such complex systems is required through advanced decision-support tools that can account for interactions and conflicts between several criteria. This leads to the need of optimizing several conflicting objectives simultaneously via reliable multicriteria decision-making models.

For the optimal design of composites, with the aid of advancement of interdisciplinary and data analysis tools, a series of criteria including mechanical, electrical, chemical, cost, life cycle assessment and environmental aspects are now able to be simultaneously considered. As one of the most efficient approach, the MCDM applications can provide the ability to formulate and systematically compare different alternatives against the large sets of design criteria. However, the mechanical behavior of woven textiles during the draping process has not been yet fully integrated to the optimal design approaches of MCDM algorithms. In this case study the criteria of mechanical behavior of the woven textile during the draping and the further involved simulations and analysis are included in the process of the optimal design and decisionmaking. For this reason the proposed optimal design strategy has been upgraded in terms of complex geometry modeling, and integration to materials selection. Comparing material properties and selecting the most appropriate materials, help to enhance the performance of products. Therefore it is important to consider and rank all the available materials.

A key objective of mechanical modeling of textiles is to define the dimensions and characteristics of a product and the materials from which it is made so that it can perform an acceptable function [73]. The area of the design decisionmaking for simultaneous consideration of the structural solution and materials selection, which is generaly needed at the early design stage is relatively weak. Although the importance of integrating materials selection and product design has been often emphasized [74].

The designer in engineering of the optimal textile structures assume a material before optimizing the geometry or select the best material for an existing geometry of a structure, but clearly either approach does not guarantee the optimal combination of geometry and material [71]. Alternatively here the materials properties are directly transmitted to the design software package so that the effect of changing materials properties on the geometry and dimentions of a component design can be directly evaluated and ranked. At the same time the engineering designer can evaluate the effect of changing geometry and dimentions on product performance.

Worth mentionning that the process of meterials selction is highly dependent on data related to material properties. In fact with a large number of materials, clearly there is a need for an information-management system [72]. Therefore in the initial proposed optimal design strategy for interactive optimization and MCDM the existing drawbacks to utilizing MCDM are improved by connecting the data mining, visualization and optimization through the user interaction and decisionmaking. Besides the materials databases are used as materials selection systems, which are essentially developed for data storage searching. Morover the electronic materials databases and data search software packages would help designer in this regard [78]

\subsection{Draping}

The manufacturing of woven reinforced composites requires a forming stage so called draping in which the preforms take the required shapes. The main deformation mechanisms during forming of woven reinforced composites are compression, bend, stretch, and shear which cause changes in orientation of the fibers. Since fiber reorientation influences the overall performance it would be an important factor that in the process of material selection to consider the draping along with the other criteria.

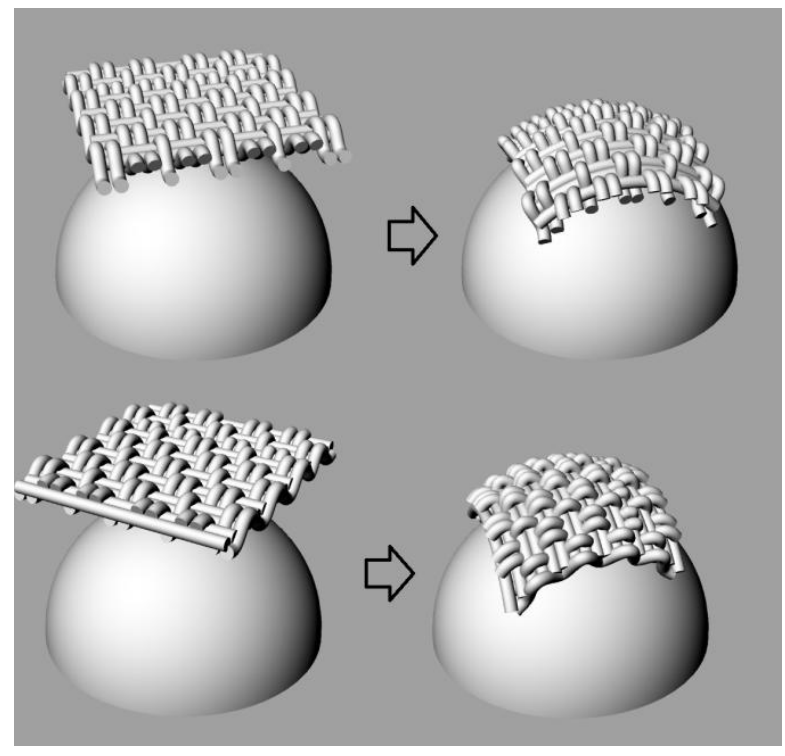

Fig 6: Simulation of draping process including a combined mechanical modeling of compression, bend, stretch, and shear shown from two different draping angles

In an optimal engineering design process for the textile composites, the materials selection integrated with draping can well determine the durability, cost, and manufacturability of final products $[61,62]$. The process would naturally involve the identification of multiple criteria properties of mechanical, electrical, chemical, thermal, environmental and life cycle costs of candidate materials [64]. In fact multiple criteria from different disciplines which are to be satisfied in a materials selection problem, often because of the criteria conflicts the complexities are even increased. Moreover the mechanical behavior of woven textiles during the draping process has not been yet fully integrated to the MCDM algorithms. Although 
many applications and algorithms of MCDM [64] have been previously presented to deal with decision conflicts often seen among design criteria in materials selection. However many drawbacks and challenges are identified associated with their applicability [67].

\subsection{Geometrical-Mechanical Modeling and Simulation of Draping}

The mechanical models of draping with a much higher computation cost, comparing to the kinematic models, offer the benefit of representing the non-linear materials behavior. Moreover the mechanical simulation, as the most promising technique, gives a real-life prediction of the fiber reorientation. Beside of all presented approaches to the geometrical modeling of woven textiles so far [68], the Spline-based methods have been the most effective technique. In fact, the Spline-based geometrical representation of a reallife model of any type of the flat-shaped woven textile, are done with implementing the related computer aided geometrical design (CAGD) code. However the mathematical representation of a multiple-dome shaped woven, which is essential for draping simulation, in the practical scale, could not be computationally efficient. Threfore in order to handle the computational complexity of geometrical modeling the multiple-dome woven shapes, utilizing the NURBS-based CAGD packages are proposed. Khabazi [70] introduced generative algorithms for creating these complex geometries. His improved algorithm is capable of producing the whole mechanism of deformation with combining all details of compressed, bended stretched and sheared properties.

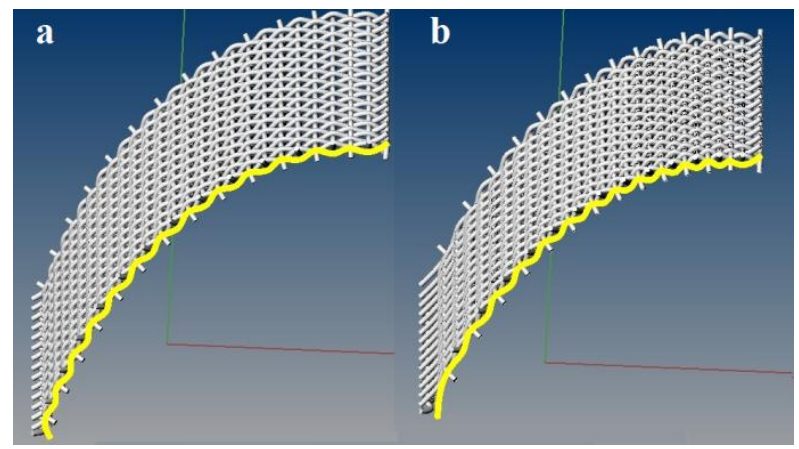

Fig 7: A combination of four different simulation criteria including the compression, bend, stretch, and shear form the draping a) Geometrical modeling and simulation of the woven textiles b) Mechanical modeling of the bending; the behavior of textile under its weight is simulated by manipulating the related geometrical model within the CAGD package.

It is assumed that if the mechanical behavior of a particular woven fabric of a particular type and material is identified then the final geometrical model of the draping could be very accurately approximated. In this technique the defined mechanical mechanisms of a particular material, in this case glass fiber [68], are translated into a geometrical logic form integrated with the NURBS-based CAGD package through the process of scripting [70].

Worh mentionning that traditionally in order to include the materials property into the mechanical models of textile the outputs from finite element analysis (FEA) are utilized as inputes to MCDM in material selection. FEA allows materials property data to be transmitted directly to a design software package so that the effect of changing materials properties on the geometry and dimentions of a component design can be directly evaluated. At the same time the DM can evaluate the effect of changing geometry and dimentions on product performance [75].

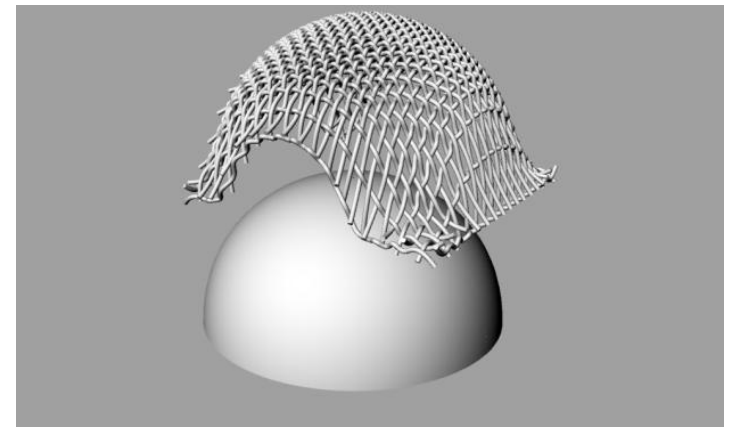

Fig 8: Geometrical modeling of double dome utilizing the Khabazi's algorithm [70].

\subsection{Integration the MCDM-Assisted Material Selection with Draping Simulation} Recently a combined FEA-MCDM approach as a framework that links the capabilities of FEA tools to the MCDM approaches for composite structural materials selection problem [75] proposed. However due do the geometricaly challenging modeling of the composite product the draping simulation has not been considered in their work.

In order to select the best material of a woven textile as well as the right angel of draping, the draping simulation needs to be carried out for a number of draping degrees for a particular material. The results of all the draping simulations of different drape angles are gathered as a data-set for consideration, in addition to already existed data-sets from the earlier case study [64], including the other criteria i.e. mechanical, electrical, chemical, cost, life cycle assessment and environmental.

\subsection{Visualization; an Effective Approach to MCDM and Material Selection}

Visualization is an effective approach in the operations research and mathematical programming applications to explore optimal solutions, and to summarize the results into an insight, instead of numbers $[65,66]$. Fortunately during past few years, it has been a huge development in combinatorial optimization, machine learning, intelligent optimization, and RSO, which have moved the research in advanced visualization methods forward [67].

The previous work in the area of visualization for MCDM [67] allows the user to better formulate the multiple objective functions for large optimization runs. Alternatively in our research utilizing RSO [79,82,83,84], which advocates learning for optimizing, the algorithm selection, adaptation and integration, are done in an automated way and the user is kept in the loop for subsequent refinements and final decisionmaking [64]. Here one of the crucial issue in MCDM is to critically analyzing a mass of tentative solutions related to materials and draping simulation, which is visually mined to extract useful information. Concerning solving the MCDM problems the DM is not distracted by technical details instead concentrates on using his expertise and informed choice among the large number of possibilities. As the whole 
process may be carried out in different design and designmaking departments worth mentioning that the workflow may overlaps with a number of other fields of research such as enterprise decision management [80].

\subsection{Software Architecture of the Reactive and Interactive MCDM Visualization Environment}

The proposed software is based on a three-tier model, independent from the optimization package $[7,8]$ which is an effective and flexible software architecture for integrating problem-solving and optimization schemes into the integrated engineering design processes and optimal design, modeling, and decision-making. The software is implemented a strong interface between the generic optimization algorithm and DM. While optimization systems produce different solutions, the DM is pursuing conflicting goals and tradeoff policies represented on the multi-dimensional graphs (see Figure 9 and $10)$.
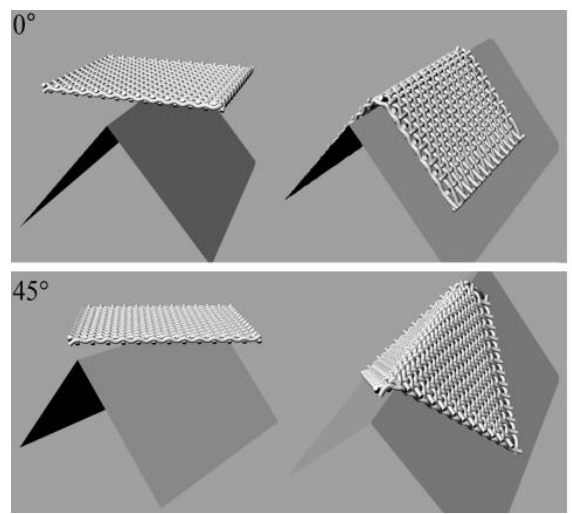
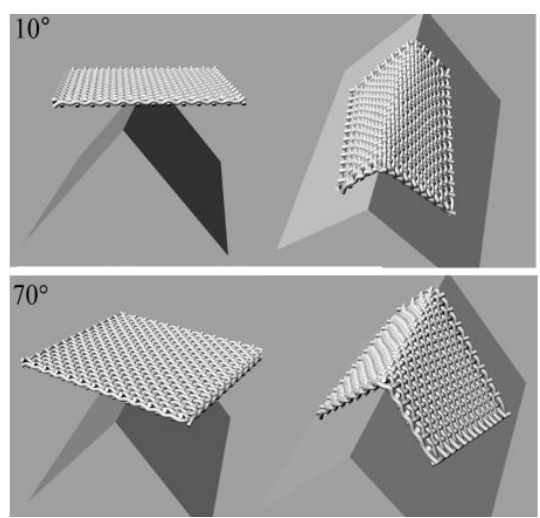
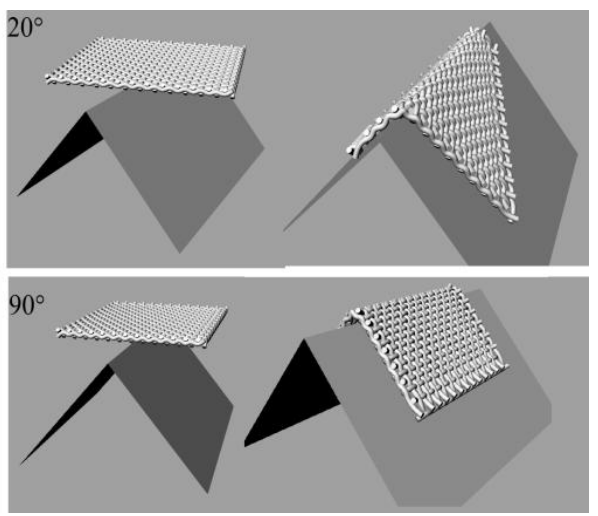

Fig 9: Mechanical modeling of draping process for a number of draping degrees
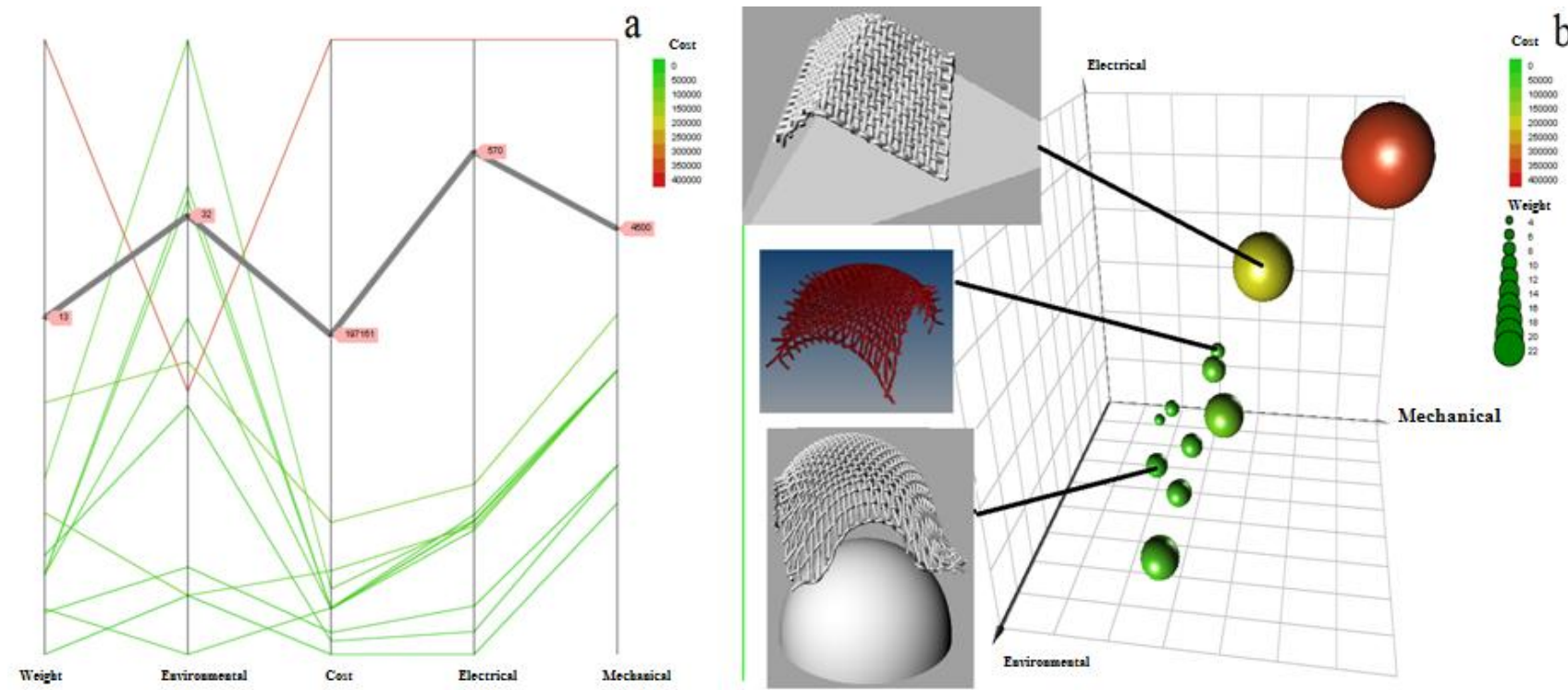

Fig 10: a) Paralel chart considering five optimization objectives simultaneously b) The 7D visualization graph used for considering different prodcuts, materials and draping characteristics simultaneously

\section{DISCUSSION AND CONCLUSIONS}

In this paper a novel environment for optimization, analytics and decision support in general engineering design problems is introduced. The utilized methodology is based on reactive search optimizationprocedure and its recently implemented software packages. The new set of powerful integrated data mining, modeling, visualiztion and learning tools via a handy procedure stretches beyond a decision-making task and attempts to discover new optimal designs relating to decision variables and objectives, so that a deeper understanding of the underlying problem can be obtained. Here along with presenting two study cases, the interactive procedure is introduced which involves the DM in the optimization process helping to choose a single solution at the end. The method is well capable of handling the big data often associated with MCDM problems.

Furthermore the preliminary tests of the software environment in the concrete context of optimal designing the welded beam 
design problem have shown the effectiveness of the approach in rapidly reaching a design preferred by the DM via advanced visualization tools and brain-computer novel interactions.

Along with presenting the latter study case the aspects of data mining, modeling, and visualization the data related to material selection are considered. Further the utilization of the proposed software architectures for multiobjective optimization and decision-making, with a particular emphasis on supporting flexible visualization is discussed. The applicability of the software can be easily customized for different problems and usage contexts.

Considering the abilities of LIONsolver and Grapheur, the interesting patterns are automatically extracted from our raw data-set via data mining tools. Additionally the advanced visual analytical interfaces are involved to support the DM interactively. With utilizing the features such as parallel filters and clustering tasks, in the material selection study case the managers can solve MOO problems as it amends previous approaches. The utilization of a software architecture for MCDM, including the mechanical modeling of draping, with a particular emphasis on supporting flexible visualization is discussed. The applicability of software can be easily customized for different problems and usage contexts.

The preliminary tests of the software environment in the concrete context of designing a multiple dome shape have shown the effectiveness of the approach in rapidly reaching a design preferred by the decision-maker.

In addition the future research is set out to investigate the role that the proposed multiobjective optimization and decisionmaking strategy can play in sustainable regional decisionmaking [86], waste management [87], and materials selection [88] in construction and demolition projects.

\section{REFERENCES}

[1] Mosavi, A. 2010. Application of Multiobjective Optimization Packages in Design of an Evaporator Coil. World Academy of Science, Engineering and Technology, V61.

[2] Esmaeili, M. and Mosavi, A. 2010. Variable reduction for multi-objective optimization using data mining techniques; application to aerospace structures, International Conference on Computer Engineering and Technology, V5, pp.333-337.

[3] Mosavi, A. 2010. The Multiobjective Optimization Package of IOSO; Applications and Future Trends, Conference of PhD students in Computer Science, CSCS, Szeged, Hungary.

[4] Mosavi, A. 2009. Computer Design and Simulation of Built Environment; Application to Forest. International Conference on Environmental and Computer Science

[5] Mosavi, A. 2009. Hydrodynamic Design and Optimization: Application to Design a General Case for Extra Equipments on the Submarine's Hull. International Conference on Computer Technology and Development, V2, pp.139-143,

[6] Mosavi, A. 2009. Parametric modeling of trees and using integrated CAD/CFD and optimization tools: Application to creating the optimal planting patterns for new forests. 2nd International Conference Wind Effects on Trees, Albert-Ludwigs-University of Freiburg, Germany.
[7] Battiti, R. and Campigotto, P. 2010. Reactive search optimization: Learning while optimizing. an experiment in interactive multiobjective optimization. In S. Voss and M. Caserta, editors, Proceedings of MIC 2009, VIII Metaheuristic International Conference, Lecture Notes in Computer Science. Springer Verlag.

[8] Battiti, R. and Brunato, M. 2011. Reactive Business Intelligence. From Data to Models to Reactive Search", Srl, Italy.

[9] Mosavi, A. 2010. On Engineering Optimization the Splined Profiles", International modeFRONTIER Users' Meeting, Trieste, Italy.

[10]Mosavi, A. 2010. The large scale system of multiple criteria decision making; pre-processing. Large Scale Complex Systems Theory and Applications, V9, Part1.

[11]Mosavi, A. 2010. Applications of Interactive Methods of MOO in Chemical Engineering Problems. Global Journals of Engineering Research, V10.

[12]Miettinen, K. and Makela, M. 1995. Interactive bundlebased method for nondifferentiable multiobjective optimization: NIMBUS. Optimization V34, 1995, 231246

[13] Goldenthal, R. and Bercovier, M. 2004. Design of curves and surfaces using multi-objective optimization.

[14]Chaudhuri, S. and Deb, K. 2010. An interactive evolutionary multi-objective optimization and decision making procedure", Applied Soft Computing, V10, 496511 .

[15]Branke, J. 2008. Consideration of Partial User Preferences in Evolutionary Multiobjective Optimization, Springer, Berlin, pp. 157-178.

[16]Tan, K.C., Lee, T.H., Khoo, D. and Khor, E.F. 2001 A multiobjective evolutionay algorithm toolbox for computer-aided multiobjective optimization", IEEE Transactions on Systems, Man and Cybernetics-Part B: Cybernetics 31 (4) 537-556.

[17]Takagi, H. 2001. Interactive evolutionary computation: fusion of the capabilities of ec optimization and human evaluation", Proceedings of IEEE 89, 1275-1296.

[18]Miettinen, K. 1999. Nonlinear Multiobjective Optimization, Kluwer, Boston.

[19]V.Chankong, Y.Y.Haimes, "Multiobjective Decision Making Theory and Methodology", North-Holland, New York, 1983.

[20]Wierzbicki, A.P. 1980. The use of reference objectives in multiobjective optimization", in: G. Fandel, T. Gal (Eds.), Multiple Criteria Decision Making Theory and Applications, Springer-Verlag, Berlin, 468-486.

[21]Kamalian, R. Takagi, H. and Agogino, A. 2004. Optimized design of mems by evolutionary multiobjective optimization with interactive evolutionary computation, in: Proceedings of the Genetic and Evolutionary Computing Conference (GECCO), 1030 1041.

[22]Deb, K. and Gupta, H. 2005. Searching for robust Paretooptimal solutions in multi-objective optimization", in: Proceedings of the Third Evolutionary Multi-Criteria Optimization (EMO-05), 150-164. 
[23]Deb, K. and Chaudhuri, S. 2007. I-MODE: an interactive multi-objective optimization and decision-making using evolutionary methods. in: Proceedings of Fourth International Conference on Evolutionary Multi-Criteria Optimization (EMO 2007), 788-802.

[24]Rekliatis, G.V., Ravindrab A., and Ragsdell, K.M. 1983. Engineering Optimisation Methods and Applications". New York: Wiley.

[25]Mosavi, A., Milani, A.S., Hoffmann, M. and Komeili, M. 2012. Multiple criteria decision making integrated with mechanical modeling of draping for material selection of textile composites. 15TH Eeuropean Conference on Composite Materials, Venice, Italy.

[26]Mosavi, A. Hoffmann, M. and Milani, A. S. 2012. Adapting the Reactive Search Optimization and Visualization Algorithms for Multiobjective Optimization Problems; Application to Geometry", Conference of PhD Students in Computer Science, Szeged, Hungary.

[27]Mosavi, A., Hoffmann, M. and Milani, A.S. 2012 Optimal Design of the NURBS Curves and Surfaces Utilizing Multiobjective Optimization and Decision Making Algorithms of RSO", Conference of PhD Students in Mathematics, Szeged, Hungary.

[28]Foldi, E., Delavar, A., Mosavi, A., Hewage, K. N. Milani, A.S., Moussavi, A.A. and Yeheyis, M.Y, 2012. Reconsidering the Multiple Criteria Decision Making Problems of Construction Projects; Using Advanced Visualization and Data Mining Tools. Conference of PhD Students in Computer Science, Szeged, Hungary, 2012.

[29]Mosavi, A., Azodinia, M., Milani, A.S., Hewage, K.N. and Yeheyis, M. 2011. Reconsidering the Multiple Criteria Decision Making Problems of Construction Workers With the aid of Grapheur", Newsletter of EnginSoft CAE Conference 2011, Year 8, No 4, Winter 2011.

[30]Mosavi, A., Azodinia, M., Milani, A.S., Hewage, K.N. and Yeheyis, M. 2011. Reconsidering the Multiple Criteria Decision Making Problems of Construction Workers With the aid of Grapheur", International ANSYS and EnginSoft Conference, Italy.

[31]Jones, C.V. 1994 Feature Article-Visualization and Optimization". INFORMS Journal on Computing, 6, 221-229.

[32]Piero, P. Subbu, R. Lizzi, J. 2009. MCDM: A framework for research and applications". IEEE Computational Intelligence Magazine. 4, pp. 48-61.

[33]Battiti, R., Brunato, M. and Mascia, F. 2008 Reactive Search and Intelligent Optimization". Operations research/Computer Science Interfaces. Springer Verlag.

[34]Mosavi, A. 2010 Multiple Criteria Decision-Making Preprocessing Using Data Mining tools. IJCSI International Journal of Computer Science Issues, Vol. 7, Issue 2, No 1.

[35] Adejuwon, A and Mosavi, A. 2010. Domain Driven Data Mining; Application to Business", IJCSI International Journal of Computer Science Issues, Vol. 7, Issue 4, No 2 .
[36]Mosavi, A. and Vaezipour, A. 2012. Reactive Search Optimization; Application to Multiobjective Optimization Problems. Applied Mathematics 3, no. 30 1572-1582.

[37]Mosavi, A. 2010 Multiobjective Optimization of Spline Curves using modeFRONTIER", International modeFRONTIER Users' Meeting, Trieste, Italy.

[38] Anzellotti, G., Battiti, R. Lazzizzera, I. Lee, P. A. Sartori, G. Soncini, G. Tecchiolli, A. Zorat, 1995. Totem: a highly parallel chip for triggering applications with inductive learning based on the reactive tabu search", In: AIHENP95. Pisa, IT

[39]Battiti, R. and Brunato, M. 2009. Reactive search optimization: learning while optimizing", In: Handbook of Metaheuristics, 2nd edn. Springer, Berlin.

[40]Battiti, R., Brunato, M. and Mascia, F. 2008. Reactive Search and Intelligent Optimization", Operations research/ Computer Science Interfaces, vol. 45. Springer Verlag.

[41]Lenne, R., Solnon, C., Stutzle, T., Tannier, E. and Birattari, M. 2008 Reactive Stochastic Local Search Algorithms for the Genomic Median Problem LECTURE NOTES IN COMPUTER SCIENCE 4972, 266.

[42]Genji, T., Oomori, T., Miyazato, K. and Hayashi, Y. N. Fukuyama, K. Co. 2003. Service Restoration in Distribution Systems Aiming Higher Utilization Rate of Feeders", In: Proc. of the Fifth Metaheuristics International Conference (MIC2003).

[43]R. Russell, W. Chiang, D. Zepeda, "Integrating multiproduct production and distribution in newspaper logistics", Computers and Operations Research 35(5), 1576-1588, 2008.

[44]Nanry, W. and Wesley Barnes, J. 2000. Solving the pickup and delivery problem with time windows using reactive tabu search", Transportation Research Part B $34(2), 107-121$

[45]Login, A. and Areas, S. 2007. Reactive tabu adaptive memory programming search for the vehicle routing problem with backhauls", Journal of the Operational Research Society 58, 1630-1641.

[46]Chambers, J. and Barnes, J. 1996. New tabu search results for the job shop scheduling problem", The University of Texas, Austin, TX, Technical Report Series ORP96-06, Graduate Program in Operations Research and Industrial Engineering.

[47]Delmaire, H. Diaz, J. Fernandez, E. and Ortega, M.1999. Reactive GRASP and Tabu Search based heuristics for the single source capacitated plant location problem", INFOR 37, 194-225.

[48]Fink, A. and Voß, S. 2003. Solving the continuous flowshop scheduling problem by metaheuristics. European Journal of Operational Research 151(2), 400-414, 2003.

[49]Potocnik, P. and Grabec, I. "Adaptive self-tuning neurocontrol", Mathematics and Computers in Simulation 51(3-4), 201-207.

[50]Winter, T. and Zimmermann, U. 2000. Real-time dispatch of trams in storage yards. Annals of Operations Research (96), 287-315. 
[51]Magdon-Ismail, M., Goldberg, M., Wallace, W. and Siebecker, D. 2003, Locating hidden groups in communication networks using hidden markov models" LECTURE NOTES IN COMPUTER SCIENCE, 126137.

[52]Hifi, M. Michrafy, M. Sbihi, A. 2006. A Reactive Local Search-Based Algorithm for the Multiple-Choice MultiDimensional Knapsack Problem. Computational Optimization and Applications 33(2), 271-285.

[53]Hu, B. and Raidl G.R. Variable neighborhood descent with self-adaptive neighborhoodordering", In: C. Cotta, A.J. Fernandez, J.E. Gallardo (eds.) Proceedings of the 7th EU/MEeting on Adaptive, Self-Adaptive, and MultiLevel Metaheuristics, malaga, Spain, 2006.

[54]Ryan, J., Bailey, T., Moore, J. and Carlton, W. 1998. Reactive tabu search in unmanned aerial reconnaissance simulations. Proceedings of the 30th conference onWinter simulation. 873-880.

[55]Kincaid, R. and Laba, K. 1998. Reactive Tabu Search and Sensor Selection inActive Structural Acoustic Control Problems", Journal of Heuristics 4(3), 199-220.

[56]Hansen, P. Mladenovic, N. 2005. Variable neighborhood search. In: E. Burke, G. Kendall (eds.) Search methodologies: introductory tutorials in optimization and decision support techniques. 211-238. Springer.

[57]Hamza, K. Mahmoud, H. Saitou, K. "Design optimization of $\mathrm{N}$-shaped roof trusses using reactive taboo search", Applied Soft Computing Journal 3(3), 221-235, 2003.

[58]Błachut, J. 2007. Tabu search optimization of externally pressurized barrels and domes", Engineering Optimization 39(8), 899-918.

[59]Mosavi, A. 2013, Data mining for decision making in engineering optimal design, Journal of Artificial Intelligence \& Data Mining, V1.

[60]Roberto, B. and Passerini, A. 2010. Brain-Computer Evolutionary Multi-Objective Optimization (BC-EMO): a genetic algorithm adapting to the decision maker. (PDF). IEEE Transactions on Evolutionary Computation 14 (15): 671-687.

[61]Ashby, M. 1999 Materials selection in mechanical design. Butterworth-Heinemann, Burlington.

[62]Jahan A., Ismail M. Y., Sapuan S. M. and Mustapha F. 2010 Material screening and choosing methods - A review. Materials and Design, 31, pp. 696-705.

[63] March, J. 1978. Bounded rationality, ambiguity, and the engineering of choice, The Bell Journal of Economics, pp. 587-608.

[64]Milani, A. S., Eskicioglu C., Robles K., Bujun K., and Hosseini-Nasab, H. 2011 Multiple criteria decision making with life cycle assessment for material selection of composites. eXPRESS Polymer Letters, 5, pp. 1062 1074.

[65]Geoffrion, A.M. 1976 The purpose of mathematical programming is insight, not numbers. Interfaces, 7, pp. 81-92.
[66]Jones, C.V. 1994 Feature Article-Visualization and Optimization. INFORMS Journal on Computing, 6, pp. 221-229.

[67]Piero P., Subbu R., Lizzi J. MCDM: A framework for research and applications. IEEE Computational Intelligence Magazine. 4, pp. 48-61(2009).

[68]Vassiliadis, S., Kallivretaki, A. and Provatidis, C., Mechanical modelling of multifilament twisted yarns. Fibers and Polymers, 11, pp. 89-96 (2010).

[69]Komeili M., and Milani A. S. Finite Element Modeling of Woven Fabric Composites at Meso-Level Under Combined Loading Modes in "Advances in Modern Woven Fabrics Technology", edited by Vassiliadis S. INTECH, Croatia, pp. 66-77 (2011).

[70]Khabazi, Z. 2010 Generative algorithms concepts and experiments weaving. Morphogenesism, London

[71]Rakshit, S. and Ananthasuresh, GK. 2008 Simultaneous material selection and geometry design of statically determinate trusses using continuous optimization. Struct Multi Optim. 35:55-68.

[72]Ashby, M. F., et al. Selection strategies for materials and processes. Materials \& Design 25.1 (2004): 51-67.

[73]Edwards KL. Selecting materials for optimum use in engineering components. Mater Des.2005;26:469-473.

[74]Edwards KL. Linking materials and design: an assessment of purpose and progress. Mater Des. $2002 ; 23: 255-264$

[75]Shanian A, Milani AS. 2012 Combined finit elementmultiple criteria optimization approach for materials selection of gas turbine component J Appl Mech.;1:302.

[76] Sirisalee P, Ashby MF, Parks GT, John Clarkson P. Multi-criteria material selection of monolithic and multimaterials in engineering design. Adv Eng Mater. 2006;8:48-56.

[77]McDowell DL, Panchal JH, Choi H-J Seepersad CC, Allen JK, Misstree F. 2010. Critical path issues in materials design. Integrated design of mutiscale, multifunctional materials and products Boston, MA: Butterworth-Heinemann, 23-38.

[78]Jahan, A. and Edwards, K.L. 2013 Multi-criteria Decision Analysis for Supporting the Selection of Engineering Materials in Product Design. Elsevier Science.

[79]Mosavi, A. 2013. A MCDM Software Tool for the Automated Design Environments. 26th Europian Conference on Operational Research, Rome.

[80] Vaezipour, A. and Mosavi, A. 2012. Enterprise Decision Management With the Aid of Advanced Business Intelligence and Interactive Visualization Tools. International CAE Conference, Verona, Italy.

[81]Mosavi, A. 2010 Multiobjective optimization package of IOSO, 24th Mini EURO Conference on Continuous Optimization and Information-Based Technologies in the Financial Sector, Izmir, Turkey.

[82]Mosavi, A. 2013 A MCDM Software Tool for Automating the Optimal Design Environments with an Application in Shape Optimization, International 
Conference on Optimization and Analysis of Structures, Tartu, Estonia, 2013.

[83]Mosavi, A. 2013. On Developing a Decision-Making Tool for General Applications to Computer Vision. International Journal of Computer Applications.

[84]Vaezipour A., et al., 2013. Machine learning integrated optimization for decision making," In Proceedings of 26th Europian Conference on Operational Research, Rome.

[85] Mosavi, A. Hoffmann, M. and Peter, N. 2009 Automatic multi-objective surface design optimisation using modeFRONTIER's CAD/CAE integrated system: Application to military submarine sail EnginSoft International Conference and ANSYS Italian Conference, Bergamo, Italy.
[86]Hossaini, N., and Hewage, K. 2013. Emergy accounting for regional studies: Case study of Canada and its provinces. Journal of environmental management, 118, 177-185.

[87] Yeheyis, M., Hewage, K., Alam, M. S., Eskicioglu, C., and Sadiq, R. 2013. An overview of construction and demolition waste management in Canada: a lifecycle analysis approach to sustainability. Clean Technologies and Environmental Policy, 15(1), 81-91.

[88]Hossaini, N., and Hewage, K. 2011. Sustainable Materials Selection for Canadian Construction Industry: An Emergy-Based Life-Cycle Analysis (Em-LCA) of Conventional and LEED Suggested Construction Materials. Journal of Sustainable Development, 5(1), p2. 